\title{
Steroids: a surgeon's view
}

\author{
J. A. R. SMITH
}

Department of Surgery, Northern General Hospital, Sheffield, England

There has been a deliberate move away from the practice of 'trying steroids' when all else fails, largely due to fear of the side-effects following such blunderbuss therapy. In surgical practice, those areas where steroids are used are now well established although it must be admitted that occasionally the proof of their value can be difficult to find.

\section{Inflammatory bowel disease}

A recent review (Lennard-Jones, 1983) has expressed disappointment at the lack of progress between knowledge of steroid pharmacology and clinical usage of these agents. A summary of current practice is provided.

\section{Ulcerative colitis}

In ulcerative colitis steroids are recommended in two distinct situations. Where the disease is limited to the distal colon and rectum, local application using either predsol enemas or hydrocortisone foam is widely practised. There is some evidence that predsol reaches more proximally when administered by enema, and clear evidence that this preparation can be absorbed producing systemic effects. Only when the disease is truly limited to the rectum on endoscopic examination can this form of therapy be used alone, i.e. without concomitant salzopyrin.

The second situation where steroid therapy is indicated is for an acute exacerbation of disease, either distal or total. The latter is of course the more serious. Steroids should then be administered intravenously, e.g. hydrocortisone $100 \mathrm{mg}$ 6-hourly as part of an aggressive regimen which includes bowel rest, intravenous fluids and frequent reassessment. Should there be any evidence of toxic megacolon despite these measures emergency total colectomy is indicated.

However, in either variety of ulcerative colitis steroids should be considered as a means of producing control of the disease process, by as short a course of therapy as feasible. There is no evidence that maintenance therapy is beneficial and considerable evidence that longer-term treatment is positively hazardous. The agent of choice for maintenance therapy is salazopyrin, which can be used safely in conjunction with any acute steroid treatment.

Correspondence: Mr F. A. R. Smith, Department of Surgery, Northern General Hospital, Herries Road, Sheffield, S5 7AU, England 
Crohn's disease

The role of steroids in Crohn's disease is now well established in theory but not always as clearly in practice.

For local disease in the anal canal or rectum, topical steriods are often valuable, as in ulcerative colitis, but should be restricted for symptomatic relief only. The macroscopic든 appearance can be much worse than the symptoms. Furthermore, Crohn's is classically a multifocal disease and often anorectal involvement will not resolve until disease elsewhere has been successfully treated, either medically or by surgery.

For systemic disease, steroids should be used only if symptomatic relief by other $\vec{\circ}$ methods is unsuccessful (e.g. control of diarrhoea) or where there is systemic disturbance. The ideal is to ensure a short course of high dosage, e.g. $15 \mathrm{mg}$ prednisolone 6-hourly, with rapid reduction in dosage as soon as a response becomes apparent. Response must be judged on clinical grounds as at the present time no satisfactory or uniform monitor of disease activity is recognized. In fact, there are a $\omega$ number of patients whose disease is too diffuse for surgery to be practicable, whow $\vec{\omega}$ require long-term maintenance therapy to achieve control of symptoms. The doseo required is variable from a modest $5 \mathrm{mg}$ daily up to $20 \mathrm{mg}$ daily and such therapy must

be accepted reluctantly in the absence of a suitable alternative.
As in ulcerative colitis, steroids in Crohn's disease should be used as seldom and for $\frac{\mathbb{D}}{\mathbb{D}}$ as short a period as possible. Steroids should also be only one part of an overal: treatment regimen.

\section{Chemotherapy}

The glucocorticoids form part of several regimens involving combination chemotherapy. The precise role of the steroids is not entirely clear but whatever else is achieved, stimulation of appetite and improvement in mood are certainly produced in a proportion of patients.

\section{Neurosurgical disease}

Dexamethasone is widely used as part of the treatment regimen for brain tumours especially of glioma type. It is believed to reduce the oedema which follows surgery ando radiotherapy. Some authorities have advocated the use of steroids to treat the oedema following severe head injury but there is little evidence of efficacy. No benefit at all was은 reported by Cooper et al. (1979) while Saul et al. (1981) reported a more complex study, in which patients were classified by a measure of their neurological dysfunction and the change in this measure produced by a standard treatment protocol. Steroids aidedo survival in those who responded to the protocol but appeared to be associated with anf increase in mortality in those who did not respond. Indeed, an alternative form of management such as fluid restriction, elective ventilation and barbiturate sedation is clearly superior. 


\section{Steroids in shock}

Greater controversy has surrounded the use of steroids in shock than their use anywhere else in surgical practice, yet it must be recognized that the supportive evidence is as strong (or as weak) as for the use of such agents for the conditions already considered. The general topic has already been reviewed (Smith, 1983) and will be summarized here.

After much discussion about adrenal cortical function in shock, a variety of conclusions were drawn (Hume \& Nelson, 1955; Frank et al., 1955; Herman et al., 1969). Initially, it was assumed that part of the shock process was due to adrenocortical failure (Nagy et al., 1964) and that adequate output of steroid was necessary to permit the action of the catecholamines. It is now clearly recognized that excessive catecholamine activity is undesirable and that steroid is not required in that category (Nickerson, 1963).

In experimental studies, hypotension and reduction in blood flow locally both caused reduction of steroid output from the adrenal gland, but administration of ACTH or restoration of flow were followed by rapid restoration of corticoid output. What is not yet clear is whether or not there is an increased requirement for steroids in shock and if so, what that requirement is. Much of this discussion was rendered superfluous when it was recognized that the role of steroid therapy in the shock syndrome was pharmacological, not simply physiological, replacement (Weil, 1962).

Many of the studies in which steroids have been claimed to be effective have had obvious flaws in design sufficient to render clinical application of the results invalid. It is hardly surprising that when physiological doses were used, a pharmacological effect was not achieved (Knapp \& Howard, 1957). Similarly, when steroids were used as 'last resort' no effect was obvious (Replogle et al., 1971), but the timing of administration of steroids as early as possible is well established experimentally (Smith \& Norman, 1979a). Equally, steroids on their own are ineffective (Raflo et al., 1975) but the need for adequate volume replacement (Smith $\&$ Norman, 1979b) and for a regimen which includes antibiotics (Hinshaw et al., 1980) are both sufficiently clear to be an accepted part of the management of severe shock.

A different objection to the use of steroids in shock has come from Ledingham $e t$ al. (1978), who claim steroid therapy is unnecessary. They have obtained results for shock resuscitation as good as those reported for steroid therapy. In a careful analysis of their experience, they reported a gradual reduction in mortality of patients admitted to the intensive care unit from $75 \%$ to $25 \%$ to $10 \%$, the last figure representing as good results as obtained anywhere by any method. However, their attitude to intensive care is quite remarkable in the intensity of the service provided and it is unlikely that such figures could be matched in general surgical or intensive care practice.

The only controlled trial is the well-established work of Schumer (1976), in which a study of patients in septic shock was reported. In both a retrospective study and a prospective study, extending over 8 years, the use of steroids as part of an overall therapeutic regimen was shown to reduce mortality in mild, moderate and severe shock. The 8-year period tends to confirm that only seriously ill patients were studied in order to recruit the members reported and while the duration of the study has now been 
criticized, the spread of annual recruitment in both control and steroid groups was similar.

Many claims have been made for the actions of steroid therapy in shock, including preservation of cellular (Spath et al., 1973) and sub-cellular (Weissman \& Thomas, 1962) integrity, prevention of the fall in 2-3 diphosphoglycerate in the red cell (McConn \& Del Guercio, 1971), pulmonary changes of shock (Brigham, 1979), avoidance of the inappropriate activation of complement by the alternate pathway (Hammerschmidt et al., 1980) and most recently, inhibition of phospholipase A2, thus preventing activation of prostoglandins and of thromboxane and prostacyclin (Lewis, 1981).

Thus, it is clear that there remain doubts about the role of steroids in severe shock but there is agreement that despite laboratory evidence demonstrating impaired mobility and phagocyte function of polymorphonuclear leucocytes, there is no evidence that pharmacological doses of steroids to a maximum of three doses of, for example, $30 \mathrm{mg}$ methylprednisolone over $\mathbf{4 8}$ hours carries any serious clinical side-effects (Lillehei et al., 1974). On balance there appears to be a case for the use of steroids on the basis that they may be beneficial and do no harm, but if used they must be in pharmacological dosage, used early and for a limited period and only as part of the full therapeutic regimen for severe shock.

\section{Steroids in pulmonary disorders}

This section will concentrate on those facets particularly relevant to the surgeon an will ignore asthma, etc.

Adult respiratory distress syndrome (ARDS) has fortunately replaced the term 'shock lung,' as the condition can be seen in a wide variety of conditions including blast trauma, decompression disease (Childs et al., 1979), fat embolus and septic shock.

Such a disparate group is difficult to study but based on the beneficial effect demonstrated in animals in gram negative septic shock in which the lung changes were reversed by methylprednisolone (Brigham, 1979) and on the reversal of the histological changes in the lungs of patients with ARDS (Wilson, 1972), the use of steroids in this condition has increased. The importance of early administration is well established experimentally (Brigham, 1979) and more evidence exists in favour of continuing use of steroids than against it, or than is available to suggest such practice is ill advised.

Thus, there are certain areas in surgical practice where steroids have a clearly defined role and others where the evidence in favour of their use marginally outbalances the contrary view, and in the absence of serious clinical side-effects future use is justified within clearly defined limits.

However, there are problems which arise in surgical practice, the incidence of which is increased by more chronic steroid therapy.

\section{Problems related to steroids in surgical practice}

Both wound infection and other septic complications occur more commonly in patients on steroid therapy and may be encouraged not only by impaired resistance to infection 
but also by the increased risk of haematoma formation, secondary to the increase in capillary fragility. Wound haematoma and infection both contribute to the impaired wound healing but in addition the glucocorticoids interfere with protein metabolism and hence with repair.

There is now a majority view that steroids do not cause peptic ulceration but that they may cause exacerbation of the ulcer and the development of complications. The most dangerous is perforation, because as in all intra-abdominal catastrophes, steroids depress the acute inflammatory response, mask the physical signs and may therefore cause a delay in management.

Patients on steroid therapy and who have been treated in the past 3-6 months may have depression of the steroid response to stress. Thus, any surgical procedure must be covered by injection of ACTH or the perioperative adminstration of hydrocortisone intravenously.

Less commonly, surgeons encounter patients on much larger doses of steroids for immune suppression after organ transplant. In addition to the above problems, there appears to be an increased risk of neoplasia, often of those types normally associated with viral induction, e.g. cervix, lip, etc. This group are at risk of infection not only from normal pathogens but also the more esoteric variety such as fungi and viruses.

\section{CONCLUSION}

In surgical practice, steroids have a clear role in many areas and a less clear but probable role in others. In short, sharp courses the associated side-effects are minimal and benefit outweighs cost. Where steroids have to be used in the longer term, certain complications occur in an incidence higher than that identified in a normal population. However, for the most part, modern surgical techniques, the use of prophylactic measures for wound healing and infection, and a greater awareness of what problems can exist have again meant that their use is better than their avoidance.

\section{REFERENCES}

Brigham K. L. (1979) Pulmonary edema: Cardiac and non cardiac. American fournal of Surgery 138, 361-7. Childs C. M., Norman J. N., Jones C., Smith J. A. R., Ross J., Riddle G., Mackintosh Allison, McKie N. I. P., McAuley I. I. \& Fructus X. (1979) Management of a complex diving accident. Undersea Biomedical Research 6, 307-16.

Cooper P. R., Moody Sarah, Clark W. K., Kirkpatrick J., Marvilla K., Gould A. L. \& Drane W. (1979) Dexamethasone and severe head injury. Fournal of Neurosurgery 51, 307-16.

Frank H. A., Frank E. D., Korman H., Macchi I. A. \& Hechter O. (1955) Corticosteroid output and adrenal blood flow during haemorrhagic shock in the dog. American fournal of Physiology 182, $24-8$.

Hammerschmidt D. E., Weaver L. J., Hudson L. D., Craddock D. R. \& Jacob H. S. (1980) Association of complement activation and elevated plasma C59 with adult respiratory distress syndrome. Lancet $\mathbf{i}, 947-9$.

Herman A. H., Mack E. A. \& Egahl R. H. (1969) Adrenal cortical secretion following prolonged haemorrhagic shock. Surgical Forum 20, 5-7.

Hinshaw L. B., Archer L. T., Beller-Todd B. K. Coalson J. J., Flournoy D. J., Passey R., Benjamin B \& 
White G. L. (1980) Survival of primates in E. coli septic shock following steroid/antibiotic therapy. fournal of Surgical Research 28, 151-70.

Hume D. M. \& Nelson D. H. (1955) Adrenal cortical function in surgical shock. Surgical Forum 5, 568-75.

Knapp R. W. \& Howard J. M. (1957) Studies on the effect of hydrocortisone on irreversible haemorrhagic shock in the dog. Surgery 42, 919-21.

Ledingham I. McA., \& McArdle C. S. (1978) Prospective study of the treatment of septic shock. Lancet ii, 1194-7.

Lennard-Jones J. E. (1983) Towards optimal use of corticosteroids in ulcerative colitis. Gut 24, 177-81.

Lewis G. P. (1981) Corticosteroids: pharmacological action and effects in trauma. Royal Society of Medicine, Forum Series 2.

Lillehei R. G., Dietzman R. H., Motsay G. J., Beckman C. B., Pomero L. H. \& Shatney C. H. (1974) Growth of the concept of shock and review of present knowledge. In Steroids and Shock Glenn T. M. (ed.) pp. 377-413. Baltimore: University Park Press.

McConn Rita \& Del Guerico L. R. M. (1971) Respiratory function of blood in the acutely ill patient and the effect of steroids. Annals of Surgery 174, 436-50.

Nagy S., Tarnoky Klara \& Petri G. (1964) Effect of a water-soluble corticosteroid analogue in experimental haemorrhagic shock. Fournal of Surgical Research 4, 62-9.

Nickerson M. (1963) Sympathetic blockade in the therapy of shock. American fournal of Cardiology 12, 619-23.

Raflo G. T., Jones R. C. W. \& Wangensteen S. L. (1975) Inadequacy of steroids in the treatment of severe haemorrhagic shock. American fournal of Surgery 130, 321-7.

Replogle R. L., Kindler H., Schottenfeld M. \& Spear S. (1971) Hemodynamic effects of dexamethasone in experiment haemorrhagic shock-negative results. Annals of Surgery 174, 126-30.

Saul T. G., Ducker T. B., Saloman M. \& Carro E. (1981) Steroids in severe head injury. fournal of Neurosurgery 54, 596-600.

Schumer W. (1976) Steroids in the treatment of clinical septic shock. Annals of Surgery 184, 333-41.

Smith J. A. R. (1983) Steroids in shock-a review. Fournal of the Royal College of Surgeons of Edinburgh 28 $214-8$.

Smith J. A. R. \& Norman J. N. (1979a) The use of glucocorticoids in refractory shock. Surgical Gynecologg and Obstetrics 149, 369-73.

Smith J. A. R. \& Norman J. N. (1979b) Volume infusion in experimental refractory shock. British fournal of Surgery 66, 80-3.

Spath J. A., Gorczynski R. J. \& Lefer, A. M. (1973) Possible mechanisms of the beneficial action of glucocorticoids in circulatory shock. Surgical Gynecology and Obstetrics 137, 597-607.

Weil M. H. (1962) The cardiovascular effects of corticosteroids. Circulation 25, 718-25.

Weissman G. \& Thomas L. (1962) Studies on lysosomes. 1. The effects of endotoxin, endotoxin tolerance and cortisone on the release of acid hydrolases from a granular fraction of rabbit liver. Fournal of Experimental Medicine 116, 433-50.

Wilson J. W. (1972) Treatment or prevention of pulmonary cellular damage with pharmacological doses of corticosteroids. Surgical Gynecology and Obstetrics 134, 675-81. 